\title{
\%
}

Francisco García Moral*

Concepción Martínez Alcalá **

Encarnación Moral Pajares**

\section{EVOLUCIÓN DE LAS EXPORTACIONES ESPAÑOLAS DE ACEITE DE OLIVA Y COMPOSICIÓN GEOGRÁFICA DE SU CRECIMIENTO (2008-2017)}

En este trabajo se analiza la evolución reciente de las exportaciones españolas de aceite de oliva que han registrado en los últimos años una intensa dinámica, consiguiendo aumentar su cuota en el total de productos agroalimentarios exportados, que pasa a ser de un 8 por 100 en 2017. En segundo lugar, se realiza un estudio geográfico del crecimiento de las ventas de este producto en el mercado mundial aplicando la metodología de shift-share. Ello permite identificar aquellos países en los que existe una demanda creciente, que presentan perspectivas favorables para las empresas oleícolas exportadoras.

Palabras clave: exportación, aceite de oliva, shift-share, España.

Clasificación JEL: F140, Q17.

\section{Introducción}

Tras la caída que experimentan las exportaciones españolas de aceite de oliva en 2009, en línea con la evolución negativa que registra en ese año las ventas de productos agrícolas y manufacturados de España en el mercado mundial, la cantidad de este producto que las empresas españolas venden en el

\footnotetext{
* Graduado en Administración y Dirección de Empresas y colaborador del Aula de Internacionalización de la Universidad de Jaén.

** Departamento de Economía de la Universidad de Jaén.

Este trabajo forma parte de los resultados de la Medida «Aulas de Internacionalización», financiada por la Consejería de Economía y Conocimiento de la Junta de Andalucía (BOJA, número 127. Miércoles, 5 de julio de 2017).

Versión de febrero de 2018.
}

mercado internacional protagoniza una intensa dinámica, pasando de $661.261,34$ toneladas en 2009 a $885.783,12$ toneladas ocho años después, registrando una tasa de variación media anual acumulada superior al 3 por 100 y consiguiendo multiplicar su cuantía por 1,34. Paralelamente, el valor de esas exportaciones crece a una tasa media anual del 10,61 por 100, consiguiendo ser de $3.456,8$ millones de euros, lo que determina que su cuota en el total de productos agroalimentarios exportados pase a ser de un 8 por 100 en 2017, cuando en 2009 representaba un 5,85 por 100.

Los objetivos de este trabajo son, en primer lugar, comparar la dinámica exportadora del $\triangleright$ 
sector del aceite de oliva ${ }^{1}$ con la operada en el sector agroalimentario nacional, tanto en términos monetarios como en valor unitario. En segundo lugar, realizar un análisis geográfico del crecimiento de las ventas de este producto en el mercado mundial aplicando la metodología de shift-share. Ello permitirá identificar aquellos países en los que existe una demanda creciente de este alimento propio de la dieta mediterránea, que viene siendo atendida satisfactoriamente por empresas españolas. La información estadística utilizada en este estudio procede de las bases de datos United Nations Commodity Trade Statistics Database (UN Comtrade) de la Organización de las Naciones Unidas y DataComex del Ministerio de Economía, Industria y Competitividad, para el periodo 2008-2017.

La metodología shift-share aplicada por Perloff, Dunn, Lampard y Muth (1960) y Dunn (1960) es una técnica utilizada mayoritariamente en el estudio del crecimiento regional, que permite desagregar la variación observada en una variable en varios componentes (shares), considerando la evolución o cambio (shifts) que presenta cada uno de ellos ${ }^{2}$. Se trata, de hecho, de cuantificar desplazamientos respecto a un comportamiento medio. La aplicación de esta técnica va a permitir analizar las exportaciones españolas de aceite de oliva y desagregar su variación total en tres efectos o causas estadísticas:

\footnotetext{
1 Identificado con la partida arancelaria 1509, que comprende:

- Aceite de oliva lampante: 15091010 (AOL).

- Aceites de oliva virgen (extra y virgen): 15091090 (AOV y AOVE). Para 2017 , los datos relativos a "aceite de oliva virgen y virgen extra» (15091090) son resultado de agregar los que se incluyen en la denominación «aceite de oliva virgen extra» (15091020) y «aceite de oliva virgen» (15091080), de acuerdo con el Reglamento de Ejecución (UE) 2017/1925 de la Comisión de 12 de octubre de 2017, por el que se modifica el anexo I del Reglamento (CEEE) n.․․ 2658/87 del Consejo relativo a la nomenclatura arancelaria y estadística y al arancel común.

- Aceite de «oliva» que contiene aceite de oliva virgen o virgen extra y aceite de oliva refinado: 15099000 (AOR).

2 Esta metodología también ha sido aplicada tradicionalmente al análisis del comercio internacional (Piezas-Jerbi y Nee, 2009).
}

(i) el efecto mundial; (ii) el efecto nacional y (iii) el efecto país, de acuerdo con la siguiente igualdad:

$$
X_{i}=m_{i}+n_{i}+p_{i}
$$

En la que $X_{i}$ es la variación de las exportaciones de aceite de oliva de España al país $i$, durante el periodo de tiempo considerado $(t)$; $m_{i}$ es la componente demanda mundial para ese mismo país; $n_{i}$ es el desplazamiento debido al comportamiento agregado de las exportaciones españolas de este producto y $p_{i}$ es el desplazamiento diferencial de cada economía nacional a la que España ha vendido aceite de oliva. Los desplazamientos diferenciales son reflejo de la especial predisposición de los distintos países a importar aceite de oliva español.

Este trabajo se estructura en cuatro apartados, incluido este de carácter introductorio. En el siguiente se realiza un estudio comparado de la evolución reciente de las exportaciones de aceite de oliva de España entre 2008 y 2017. El apartado tercero recoge un análisis shiftshare por países, que permite identificar los mercados exteriores en los que la demanda de aceite de oliva español ha mantenido una intensa dinámica, lo que ha contribuido a la expansión de las ventas extranacionales del sector en los últimos años. Por último, el apartado cuarto refiere brevemente las reflexiones finales.

\section{Evolución de las exportaciones de aceite de oliva}

En el Gráfico 1 se compara la evolución de las exportaciones de aceite de oliva de España y las que protagoniza el sector agroalimentario $D$ 
GRÁFICO 1

EVOLUCIÓN DE LAS EXPORTACIONES ESPAÑOLAS DE ACEITE DE OLIVA Y DEL SECTOR AGROALIMENTARIOY CUOTA DE LAS EXPORTACIONES DE ACEITE DE OLIVA EN EL TOTAL DEL SECTOR AGROALIMENTARIO ENTRE 2008 Y 2017

(En porcentaje)

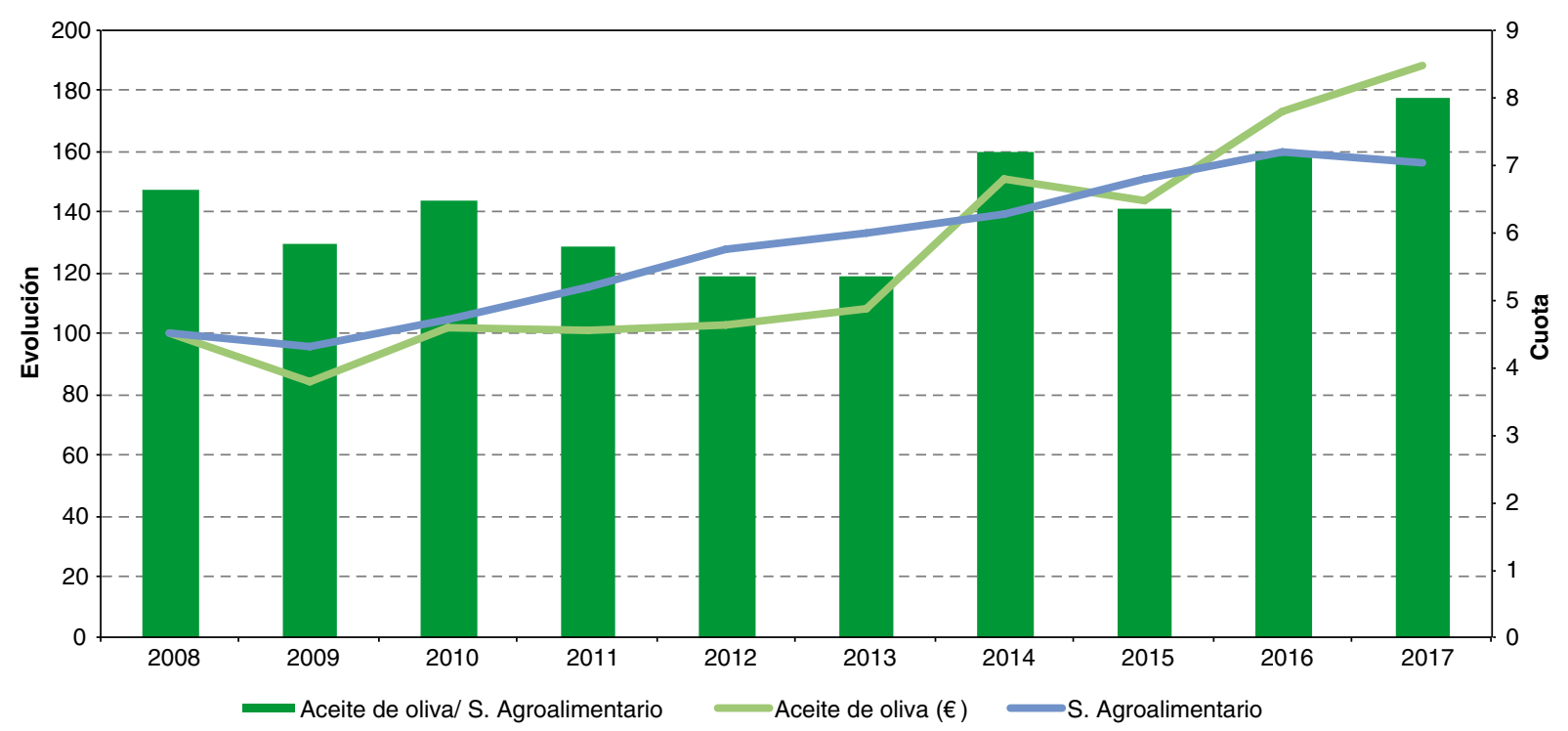

Fuente: DataComex. Elaboración propia.

nacional ${ }^{3}$ entre 2008 y 2017. Además, se representa la cuota de este producto en el total exportado por el sector agroalimentario en cada uno de los años. Los datos representados confirman varios hechos. En primer lugar, el crecimiento constante de las ventas de aceite de oliva realizadas en el mercado internacional, que consiguen elevar su valor a más de tres mil millones de euros, tanto en 2016 como en 2017. En segundo término, que si bien el proceso seguido es continuado en el tiempo, registra una dinámica dispar, más pausada entre 2008 y 2013, acelerándose de forma intensa en los últimos años del periodo analizado. En el primer subperiodo, 2008-2013, la tasa de variación media anual acumulada que registran las exportaciones de aceite de oliva es de un 1,56 por 100 , cuando la que corresponde al sector agroalimentario

3 Identificado con los 24 primeros capítulos arancelarios, de acuerdo con la Subdirección General de Comercio Internacional de Productos Agroalimentarios (2017). asciende a un 5,96 por 100. Muy al contrario, en la segunda etapa, las ventas del subsector oleícola alcanzan una variación media anual acumulada del 14,93 por 100 , superior a la imputable al conjunto de productos agroalimentarios, que asciende a un 4,02 por 100 .

Paralelamente al proceso descrito, la información estadística representada en el Gráfico 2 permite constatar el incremento observado en el valor unitario (euros $/ \mathrm{kg}$ ) del aceite de oliva exportado, que se sitúa en 3,90 euros en 2017, un 39,16 por 100 más que en 2008, y presentando una variación positiva de más de un euro entre 2014 y 2017, subperiodo en el que se produce el mayor incremento. El valor unitario imputable al conjunto de los productos agroalimentarios exportados registra, sin embargo, una menor variación, pasando de 1,10 euros en 2008 a 1,38 euros en 2017. La evolución seguida por el valor unitario del aceite responde, en gran parte, al aumento del consumo $y, \triangleright$ 


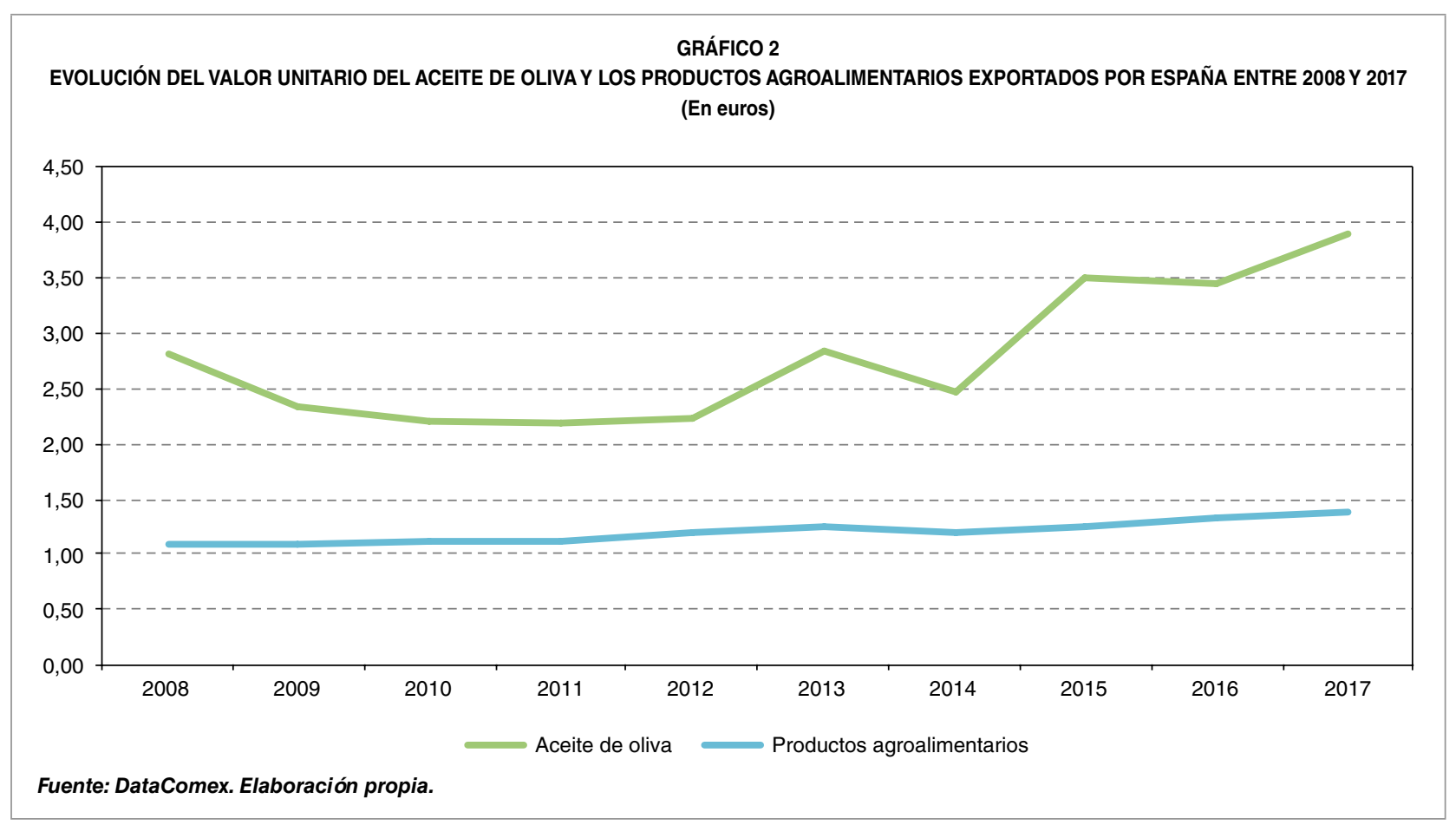

concretamente, en países que tradicionalmente no han sido importantes demandantes de esta grasa vegetal (Moral y Lanzas, 2017), que han pasado de representar el 18,47 por 100 del total mundial en la campaña 2000/2001 a un 28,35 por 100 en la campaña 2017/2018, según el Consejo Oleícola Internacional (COI). Frente a esta situación, la oferta permanece estable en el corto y medio plazo, dadas las características productivas del olivar y su dependencia de las condiciones climatológicas.

En los últimos años, Italia, que siempre ha presentado un consumo elevado en volumen, observa fuertes caídas a partir de la campaña 2006/2007, hasta alcanzar niveles de 514.000 toneladas diez años después, valores muy bajos y similares a las cifras imputables a España. Grecia, al igual que Italia, registra una fuerte reducción en sus compras, que viene explicada por los años de crisis económica del país. Por el contrario, países no mediterráneos como Estado Unidos, Japón, China o India protagonizan aumentos importantes en el consumo, que claramente se traducen en compras realizadas en el mercado internacional, al tratarse de economías con una escasa o nula producción nacional, incapaz de satisfacer la demanda interna. Mercados que, según el COI, presentan margen de crecimiento.

Atendiendo a la categoría del producto, el Cuadro 1 muestra la distribución de las exportaciones entre aceite de oliva lampante ( $A O L)$, aceite de oliva virgen y virgen extra (AOV+AOVE) $y$ aceite de «oliva» (AOR), mezcla de aceite de oliva refinado y aceite de oliva virgen o virgen extra, y el valor unitario por categorías en los diferentes años. El sector vende sobre todo aceite de oliva virgen y virgen extra, que representa algo menos de tres cuartas partes del total en el conjunto del periodo. Esta variedad es la que mayor valor unitario presenta, a excepción de 2008, siendo, además, la que experimenta una variación más pronunciada, al aumentar 1,16 euros en los nueve años considerados.

El aceite de «oliva» (AOR) mantiene una cuota media del 22,40 por 100 en volumen y $\triangleright$ 
EVOLUCIÓN DE LAS EXPORTACIONES ESPAÑOLAS DE ACEITE DE OLIVA Y COMPOSICIÓN...

CUADRO 1

VALOR UNITARIO DEL ACEITE DE OLIVA LAMPANTE (AOL), VIRGEN Y VIRGEN EXTRA (AOV+AOVE) Y ACEITE DE «OLIVA» (AOR) EXPORTADO Y CUOTA DE CADA CATEGORÍA EN EL TOTAL EXPORTADO ENTRE 2008 Y 2017

(En porcentaje)

\begin{tabular}{|c|c|c|c|c|c|c|c|c|c|c|c|}
\hline & 2008 & 2009 & 2010 & 2011 & 2012 & 2013 & 2014 & 2015 & 2016 & 2017 & 2008-2017 \\
\hline \multicolumn{12}{|c|}{ Valor unitario (euros/kg) } \\
\hline AOL & 2,39 & 1,84 & 1,92 & 1,61 & 1,82 & 2,48 & 1,98 & 2,83 & 3,00 & 3,30 & 2,32 \\
\hline $\mathrm{AOV}+\mathrm{AOVE}$ & 2,79 & 2,37 & 2,28 & 2,24 & 2,29 & 2,84 & 2,53 & 3,63 & 3,50 & 3,95 & 2,84 \\
\hline AOR & 2,91 & 2,34 & 1,99 & 2,15 & 2,14 & 2,83 & 2,35 & 3,28 & 3,32 & 3,89 & 2,72 \\
\hline \multicolumn{12}{|c|}{ Cuota en euros } \\
\hline AOL & 3,40 & 5,09 & 4,27 & 4,20 & 2,12 & 2,63 & 4,96 & 4,74 & 2,53 & 4,75 & 3,90 \\
\hline $\mathrm{AOV}+\mathrm{AOVE}$ & 73,12 & 73,14 & 75,27 & 75,12 & 75,26 & 75,51 & 74,67 & 73,23 & 75,35 & 72,36 & 74,23 \\
\hline AOR & 23,49 & 21,77 & 20,46 & 20,68 & 22,62 & 21,87 & 20,37 & 22,04 & 22,12 & 22,89 & 21,87 \\
\hline \multicolumn{12}{|c|}{ Cuota en toneladas } \\
\hline $\mathrm{AOL}$ & 3,98 & 6,45 & 4,88 & 5,70 & 2,60 & 2,99 & 6,15 & 5,87 & 2,90 & 5,61 & 4,75 \\
\hline$A O V+A O V E$ & 73,38 & 71,89 & 72,52 & 73,25 & 73,69 & 75,16 & 72,58 & 70,58 & 74,19 & 71,43 & 72,86 \\
\hline AOR & 22,64 & 21,66 & 22,59 & 21,06 & 23,71 & 21,84 & 21,28 & 23,55 & 22,90 & 22,96 & 22,40 \\
\hline
\end{tabular}

de un 21,87 por 100 en valor, registrando en términos comparativos un valor promedio alto, solo 12 céntimos inferior al que corresponde al oliva virgen y virgen extra, lo que puede responder a factores relacionados con el conocimiento que el comprador tenga del producto, no familiarizado con los atributos sensoriales del aceite de oliva virgen y virgen extra, incapaz de apreciar el color y el sabor del aceite de más alta calidad. Tal y como sostienen Sayadi, Erraach y Parra-López (2017), los consumidores están confundidos y desinformados en la interpretación de los signos de calidad del AOR y el AOV y AOVE, lo que exige una intensa labor de información y formación al consumidor que permita al demandante percibir la diferencia y conocer lo que le ofrece cada variedad. El aceite lampante, no consumible directamente, es al que le corresponde un menor valor unitario.

Por países, los datos del Cuadro 2 permiten advertir ciertas diferencias en las preferencias de los importadores, en función del país de origen. Francia compra sobre todo AOV y AOVE, que representa más del 90 por 100 del total de sus importaciones a España. En segundo lugar, China es la que presenta una mayor cuota para el AOV y AOVE, aunque el AOR ha ido ganando posiciones. Italia, con un consumo per cápita de 9,2 kg, adquiere mayoritariamente AOV y AOVE, habiendo reducido progresivamente la cuota del AOR, en favor principalmente del AOL. En Estado Unidos el porcentaje que corresponde al AOV y AOVE es mucho más reducido, en términos comparativos, suponiendo menos del 50 por 100 en el conjunto del periodo, siendo elevada la cuota que corresponde al aceite de menor calidad.

Los datos publicados por la base DataComex relativos a 2017 permiten conocer, por primera vez, qué porcentaje de aceite de oliva virgen y virgen extra se exporta en envases de menos de 5 litros. De nuevo, esta información constata políticas de comercialización muy dispares, en función del país de destino, pues mientras que un 54,72 y un 56,83 por 100 del aceite de oliva virgen extra exportado a Estados Unidos y China, respectivamente, se envasa en este tipo de recipiente, en Italia no se vende nada en estos envases y en Francia solo un 0,8 por 100 . $\triangleright$ 
Francisco García Moral, Concepción Martínez Alcalá y Encarnación Moral Pajares

CUADRO 2

CUOTA DEL AOL, AOV+AOVE Y AOR EN EL TOTAL EXPORTADO A ITALIA, FRANCIA, ESTADOS UNIDOS Y CHINA ENTRE 2008 Y 2017 (En porcentaje)

\begin{tabular}{|c|c|c|c|c|c|c|c|c|c|c|c|}
\hline & 2008 & 2009 & 2010 & 2011 & 2012 & 2013 & 2014 & 2015 & 2016 & 2017 & 2008-2017 \\
\hline \multicolumn{12}{|c|}{ Italia } \\
\hline $\mathrm{AOL}$ & 8,54 & 12,75 & 10,27 & 11,89 & 6,72 & 7,58 & 13,33 & 15,85 & 7,11 & 14,58 & 11,06 \\
\hline$A O V+A O V E$ & 71,51 & 70,78 & 70,62 & 74,42 & 72,59 & 76,78 & 70,90 & 69,33 & 74,32 & 67,51 & 71,75 \\
\hline $\mathrm{AO}$ & 19,95 & 16,47 & 19,10 & 13,69 & 20,69 & 15,64 & 15,77 & 14,81 & 18,57 & 17,91 & 17,19 \\
\hline \multicolumn{12}{|c|}{ Francia } \\
\hline $\mathrm{AOL}$ & 2,48 & 0,77 & 0,04 & 0,05 & 0,31 & 0,21 & 0,79 & 1,37 & 0,72 & 0,69 & 0,74 \\
\hline$A O V+A O V E$ & 96,87 & 98,18 & 88,70 & 99,02 & 97,77 & 97,92 & 97,45 & 95,05 & 95,39 & 95,43 & 96,15 \\
\hline AOR & 0,65 & 1,05 & 11,25 & 0,93 & 1,93 & 1,87 & 1,76 & 3,59 & 3,89 & 3,88 & 3,11 \\
\hline \multicolumn{12}{|c|}{ Estados Unidos } \\
\hline AOL & 0,00 & 0,69 & 0,00 & 0,54 & 0,00 & 0,06 & 0,15 & 0,00 & 0,00 & 0,00 & 0,12 \\
\hline$A O V+A O V E$ & 52,19 & 66,50 & 72,35 & 49,32 & 31,17 & 56,69 & 43,17 & 6,90 & 46,60 & 45,58 & 46,38 \\
\hline AOR & 47,81 & 32,81 & 27,65 & 50,15 & 68,83 & 43,26 & 56,69 & 93,09 & 53,40 & 54,42 & 53,50 \\
\hline \multicolumn{12}{|c|}{ China } \\
\hline $\mathrm{AOL}$ & 0,32 & 0,21 & 0,07 & 0,30 & 0,07 & 0,00 & 0,03 & 0,00 & 0,00 & 0,00 & 0,06 \\
\hline$A O V+A O V E$ & 94,16 & 94,51 & 86,58 & 93,42 & 92,88 & 87,21 & 76,39 & 71,97 & 80,68 & 80,72 & 84,06 \\
\hline AOR & 5,53 & 5,28 & 13,35 & 6,28 & 7,04 & 12,78 & 23,58 & 28,03 & 19,31 & 19,28 & 15,87 \\
\hline
\end{tabular}

GRÁFICO 3

NÚMERO DE EMPRESAS ESPAÑOLAS EXPORTADORAS DE ACEITE DE OLIVA (CNAE 1014), REGULARES Y NO REGULARES, Y PORCENTAJE QUE REPRESENTAN LAS VENTAS DE LAS 25 PRIMERAS COMPAÑÍAS EXPORTADORAS DEL SECTOR OLEÍCOLA

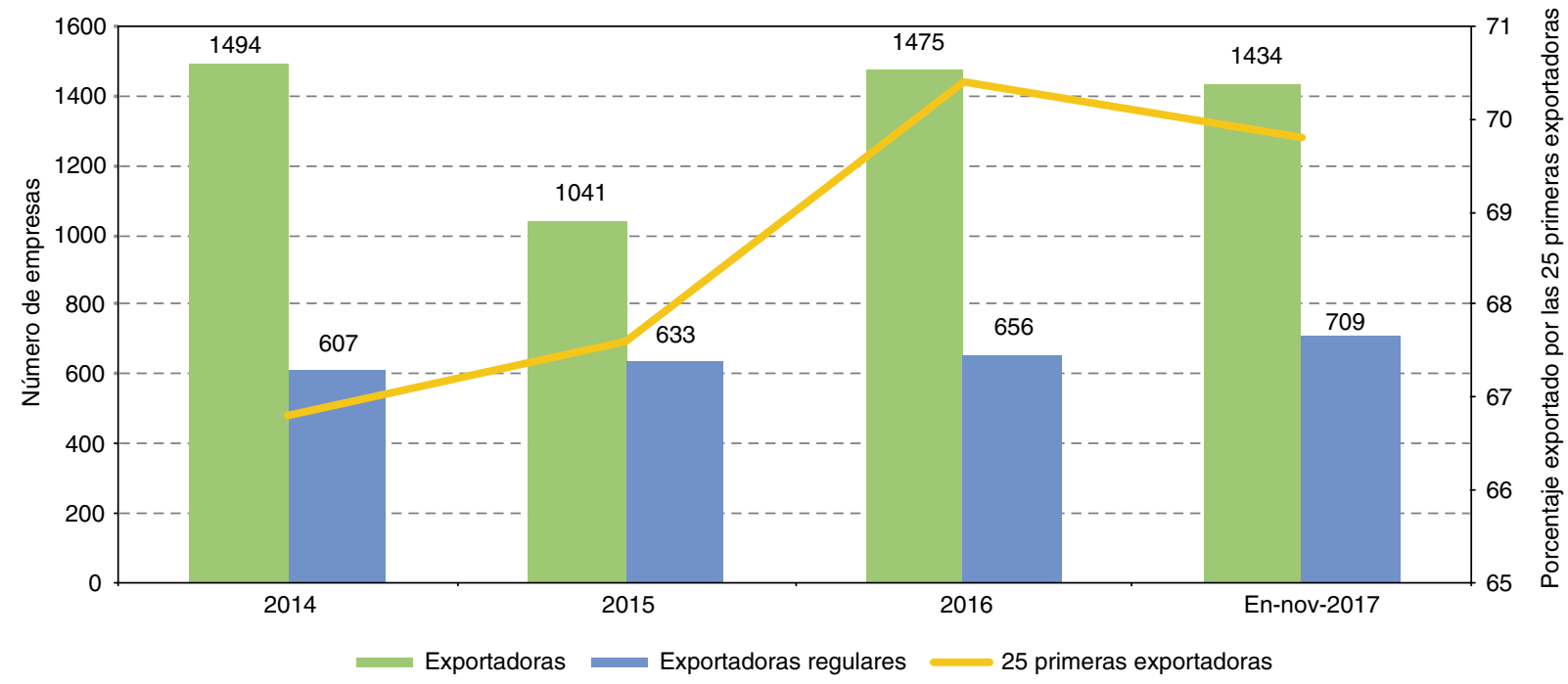

*Empresas regulares son las que han exportado durante los últimos cuatro años consecutivos (ICEX, 2018).

Fuente: Perfil de la empresa exportadora española, ICEX. Elaboración propia.

Por último, conviene tener presente que la actividad exportadora de un país es el resultado de lo que hacen sus empresas. La información que presenta el Gráfico 3 permite constatar que, aunque son muchas las compañías del sector que cada año colocan parte de su producción en el mercado internacional, más de mil, solo un porcentaje que oscila $\triangleright$ 
entre un 40,63 por 100 en 2014 y un 60,81 por 100 en 2015, acuden regularmente al mismo. No obstante, el número de este tipo de compañías se va consolidando poco a poco, llegando a ser más de 700 en 2017. Son una minoría, sin embargo, las que concentran más del 60 por 100 del total vendido, porcentaje que se eleva a un 70,4 por 100 en 2016. Tal y como refieren Moral y Lanzas (2014) para el caso de Andalucía, la mayoría de las empresas del sector son de pequeña dimensión y acuden a los mercados internacionales de forma esporádica y ocasional o simplemente atienden la demanda de clientes extranjeros interesados por el producto. Estas compañías, exportadoras pasivas, no cuentan con un departamento de exportación ni una estrategia claramente definida para su internacionalización.

\section{Factores geográficos en el crecimiento de las exportaciones}

Para conocer cómo la geografía de las exportaciones españolas de aceite de oliva ha influido en la evolución reciente de la variable y, concretamente, entre 2013 y 2017, subperiodo en el que tiene lugar una gran expansión del negocio internacional, el Cuadro 3 presenta la distribución por países de las ventas en el exterior y los resultados del análisis shift-share realizado.

Destaca, ante todo, el elevado grado de concentración de las ventas, ya que solo un país, Italia, es destino de un 38,20 y un 34,24 por 100 de lo exportado en los años de referencia, lo que hace a la industria oleícola española muy dependiente de la demanda procedente de este socio comunitario. Una demanda que, en parte, responde a las necesidades de su mercado interno, que no puede ser satisfecha con su producción nacional ${ }^{4} \mathrm{y}$, en parte, tiene carácter intraindustrial, obedeciendo a los pedidos de clientes internacionales a los que las empresas italianas venden aceite de oliva. Conviene tener en cuenta, sin embargo, que esta economía ha reducido su peso en favor de terceros mercados.

En una posición intermedia, con una cuota entre un 8 y un 14 por 100 figuran Estados Unidos, Francia y Portugal, que mantienen dinámicas dispares, pues mientras que el mercado norteamericano incrementa considerablemente sus importaciones de aceite de oliva procedente de España en estos años, llegando a sumar 432,22 millones de euros como media entre 2016 y 2017, un 13,02 por 100 del total, Francia prácticamente mantiene su cuota y Portugal, que también ha aumentado sus compras, la reduce. Un tercer grupo está conformado por Reino Unido, China y Japón, con importaciones en 2016 y 2017 superiores a los 100 millones de euros. Australia, Alemania, Brasil, Países Bajos, México, Corea del Sur y Bélgica configuran un cuarto agregado, responsable en conjunto de un 10,23 por 100 del total exportado en 2016-2017. Por último, se incluye un variado agregado de países, concretamente 26 , muy dispares entre sí, tanto en relación con su nivel de desarrollo como en su posición geográfica, en los que las empresas españolas protagonizaron operaciones de exportación por una cuantía inferior a 27 millones de euros en 2017.

A partir de la información recogida en la columna tercera del Cuadro 3, que presenta la $D$

\footnotetext{
4 Según los datos publicados por el COI, el consumo estimado para Italia en la campaña $2016 / 2017$ es de 514.000 toneladas y su producción en esa misma campaña asciende a 182.300 toneladas. Aunque tradicionalmente este país ha mantenido una producción inferior a su demanda, la aparición de la bacteria Xylella fastidiosa en octubre de 2014 ha mermado considerablemente su potencial productivo. Los datos sobre producción pueden consultarse en http://www.internationaloliveoil.org/ estaticos/view/131-world-olive-oil-figures?lang=es_ES
} 
CUADRO 3

DISTRIBUCIÓN POR PAÍSES DE LAS EXPORTACIONES DE ACEITE DE OLIVA DE ESPAÑA EN 2013-2014 Y 2016-2017 Y ANÁLISIS SHIFT-SHARE DE LAS EXPORTACIONES DE ACEITE DE OLIVA

(En porcentaje)

\begin{tabular}{|c|c|c|c|c|c|c|}
\hline \multirow{2}{*}{ País } & \multicolumn{2}{|c|}{ Distribución por países ${ }^{1}$} & \multicolumn{4}{|c|}{ Análisis shift-share (2013-2014)-(2016-2017) } \\
\hline & 2013-2014 & 2016-2017 & Variación total & Efecto mundial ${ }^{2}$ & Efecto nacional ${ }^{2}$ & Efecto país ${ }^{2}$ \\
\hline 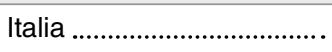 & 38,20 & 34,24 & 27,90 & 42,46 & 110,60 & $-53,05$ \\
\hline Estados Unidos .................... & 9,25 & 13,02 & 100,93 & 11,74 & 30,58 & 57,68 \\
\hline 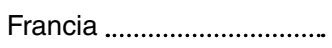 & 9,52 & 9,51 & 42,65 & 27,78 & 72,36 & $-0,13$ \\
\hline Portugal .. & 10,58 & 8,84 & 19,24 & 61,56 & 160,36 & $-121,93$ \\
\hline Reino Unido ... & 4,60 & 4,50 & 39,39 & 30,07 & 78,34 & $-8,42$ \\
\hline China & 3,15 & 3,99 & 80,63 & 14,69 & 38,27 & 47,03 \\
\hline Japón & 3,10 & 3,62 & 66,76 & 17,75 & 46,23 & 36,02 \\
\hline 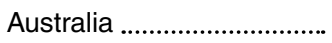 & 2,24 & 2,22 & 41,74 & 28,39 & 73,95 & $-2,33$ \\
\hline Alemania ....... & 1,14 & 1,85 & 131,88 & 8,98 & 23,40 & 67,62 \\
\hline 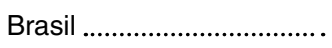 & 2,30 & 1,44 & $-10,93$ & $-108,36$ & $-282,26$ & $-490,62$ \\
\hline 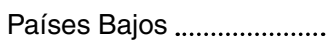 & 1,42 & 1,36 & 36,53 & 32,43 & 84,48 & $-16,91$ \\
\hline 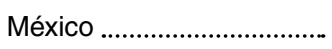 & 1,29 & 1,23 & 35,88 & 33,02 & 86,01 & $-19,03$ \\
\hline 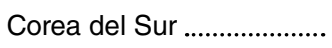 & 1,03 & 1,13 & 55,98 & 21,16 & 55,13 & 23,70 \\
\hline Bélgica ................... & 1,15 & 1,00 & 23,48 & 50,45 & 131,43 & $-81,88$ \\
\hline Rusia .................. & 1,68 & 0,83 & $-29,42$ & $-40,27$ & $-104,89$ & $-245,16$ \\
\hline 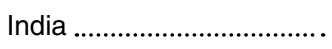 & 0,62 & 0,72 & 66,28 & 17,87 & 46,56 & 35,56 \\
\hline 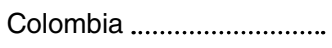 & 0,59 & 0,56 & 36,10 & 32,82 & 85,48 & $-18,30$ \\
\hline 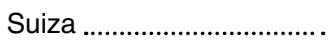 & 0,51 & 0,55 & 52,00 & 22,78 & 59,35 & 17,87 \\
\hline 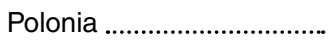 & 0,34 & 0,48 & 100,64 & 11,77 & 30,67 & 57,56 \\
\hline 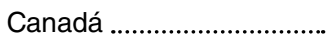 & 0,20 & 0,42 & 202,81 & 5,84 & 15,22 & 78,94 \\
\hline 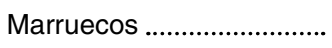 & 0,33 & 0,42 & 83,78 & 14,14 & 36,83 & 49,03 \\
\hline 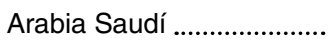 & 0,49 & 0,40 & 16,92 & 70,00 & 182,36 & $-152,36$ \\
\hline 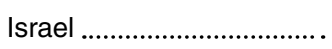 & 0,35 & 0,38 & 57,57 & 20,58 & 53,61 & 25,81 \\
\hline 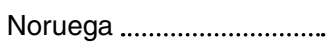 & 0,32 & 0,34 & 54,04 & 21,92 & 57,11 & 20,96 \\
\hline Suecia .......... & 0,26 & 0,34 & 84,02 & 14,10 & 36,73 & 49,17 \\
\hline República Dominicana ..... & 0,32 & 0,32 & 42,63 & 27,79 & 72,40 & $-0,19$ \\
\hline 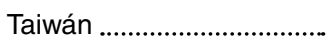 & 0,31 & 0,30 & 38,50 & 30,77 & 80,16 & $-10,93$ \\
\hline Austria & 0,21 & 0,30 & 97,88 & 12,10 & 31,53 & 56,37 \\
\hline 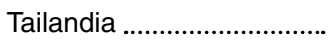 & 0,32 & 0,28 & 24,62 & 48,12 & 125,36 & $-73,48$ \\
\hline República Checa ................... & 0,19 & 0,24 & 80,72 & 14,68 & 38,23 & 47,09 \\
\hline Nueva Zelanda ...................... & 0,22 & 0,24 & 57,42 & 20,63 & 53,75 & 25,62 \\
\hline 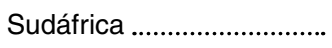 & 0,18 & 0,22 & 79,73 & 14,86 & 38,71 & 46,43 \\
\hline 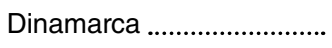 & 0,18 & 0,22 & 73,76 & 16,06 & 41,84 & 42,09 \\
\hline 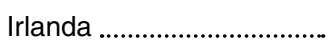 & 0,31 & 0,21 & $-3,39$ & $-349,88$ & $-911,42$ & $-1361,30$ \\
\hline 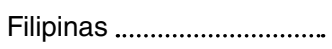 & 0,10 & 0,21 & 208,49 & 5,68 & 14,80 & 79,52 \\
\hline 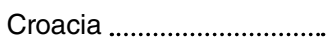 & 0,10 & 0,19 & 177,37 & 6,68 & 17,40 & 75,92 \\
\hline 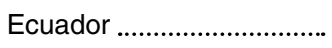 & 0,20 & 0,18 & 29,36 & 40,36 & 105,12 & $-45,48$ \\
\hline Emiratos Árabes Unidos .. & 0,31 & 0,17 & $-19,95$ & $-59,38$ & $-154,67$ & $-314,04$ \\
\hline Finlandia & 0,16 & 0,17 & 52,06 & 22,76 & 59,28 & 17,97 \\
\hline 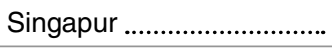 & 0,16 & 0,13 & 21,86 & 54,19 & 141,15 & $-95,34$ \\
\hline \multicolumn{7}{|c|}{$\begin{array}{l}\text { (1) El agregado de países considerado concentra el } 97,72 \text { y } 96,78 \text { por } 100 \text { de las exportaciones de aceite de oliva de España en 2013-2014 y 2016-2017, } \\
\text { respectivamente. }\end{array}$} \\
\hline \multicolumn{7}{|c|}{ (2) Porcentaje sobre la variación total. } \\
\hline \multicolumn{7}{|c|}{ Fuente: Comtrade y DataComex. Elaboración propia. } \\
\hline
\end{tabular}


variación total de las exportaciones de aceite de oliva por países entre el año inicial (promedio de 2013-2014) y el final (promedio de 2016$2017)^{5}$, los países que más han incrementado sus importaciones son Filipinas (208,49 por $100)$ y Canadá $(202,81$ por 100$)$, dos economías muy dispares entre sí, con pautas alimenticias muy diferentes a las que presentan los países mediterráneos, en los que el aceite de oliva es parte de su dieta diaria. Con variaciones superiores al 100 por 100 figuran Croacia (177,37 por 100), Alemania (131,88 por 100), Estados Unidos (100,93 por 100) y Polonia (100,64 por 100). Existen, además, quince mercados nacionales en los que las exportaciones registran un incremento superior al 42,71 por 100 imputable al conjunto de las ventas de aceite de oliva de España. Estos países son: Austria (97,88 por 100), Marruecos (83,78 por 100$)$, República Checa $(80,72$ por 100$)$, China $(80,63$ por 100), Suecia (84,02 por 100), Sudáfrica (79,730 por 100), Dinamarca (73,76 por 100$)$, Japón (66,76 por 100), India (66,28 por 100), Israel (57,57 por 100), Nueva Zelanda (57,42 por $100)$, Corea del sur $(55,98$ por 100$)$, Noruega $(54,04$ por 100), Finlandia (52,06 por 100) y Suiza (52,00 por 100). Frente a esta evolución sorprende la caída en las compras que protagonizan Brasil, Rusia, Irlanda y Emiratos Árabes Unidos, que ven reducida su cuota.

En las tres columnas restantes del Cuadro 3 se registra, para cada país, en qué porcentaje cada uno de los tres efectos considerados en el análisis shift-share explica la variación agregada. Así, para Estados Unidos, el dinamismo de la demanda de aceite de oliva a nivel mundial explica el 11,74 por 100 de la variación total, mientras que el efecto nacional asciende a

5 De acuerdo con Donoso y Martín (2007) y Seguí y Monserrat (2008), consideramos que el análisis shift-share debe hacerse a partir del valor promedio de dos años, tanto al inicio como al final del periodo de estudio. un 30,58 por 100 , siendo el efecto país de un 57,68 por 100. Paralelamente, en China, el efecto demanda mundial es de un 14,69 por 100; el nacional, de un 38,27 por 100 y de un 47,03 por 100 el efecto país.

A excepción de las economías que han reducido sus importaciones en este subperiodo, el análisis particularizado del efecto país permite identificar dos patrones que responden a diferencias significativas. Un primer grupo incluye quince mercados en los que el efecto país es negativo, pero en los que el efecto demanda mundial es elevado, superior al 27 por 100 , presentando, asimismo, un efecto nacional positivo. Cuatro de estos destinos son Italia, Francia, Portugal y Reino Unido, mercados consolidados, en los que el aceite de oliva español mantiene buenas cuotas de exportación y en los que el efecto nacional permite advertir cierto margen para el crecimiento de las ventas. Completan este grupo once economías muy heterogéneas entre sí, con unas compras en 2017 inferiores a 70 millones de euros, entre los que se encuentran tres países europeos (Países Bajos, Bélgica, Austria), cuatro de América Latina (México, Colombia, República Dominicana y Ecuador) y cinco de Asia y Oceanía (Australia, Arabia Saudí, Taiwán, Tailandia y Singapur). En conjunto, son destinos que importan aceite de oliva procedente de España, en los que existe una demanda local satisfecha por las compras realizadas en el mercado internacional y en los que las firmas de las distintas regiones españolas colocan una parte de su producción, pero que en los últimos años no se muestran lo suficientemente dinámicos.

Un segundo grupo incluye veinte economías nacionales, todas ellas con un efecto país positivo. En estas, la demanda de aceite de oliva con origen en España ha seguido una tendencia creciente, registrando las exportaciones $\triangleright$ 


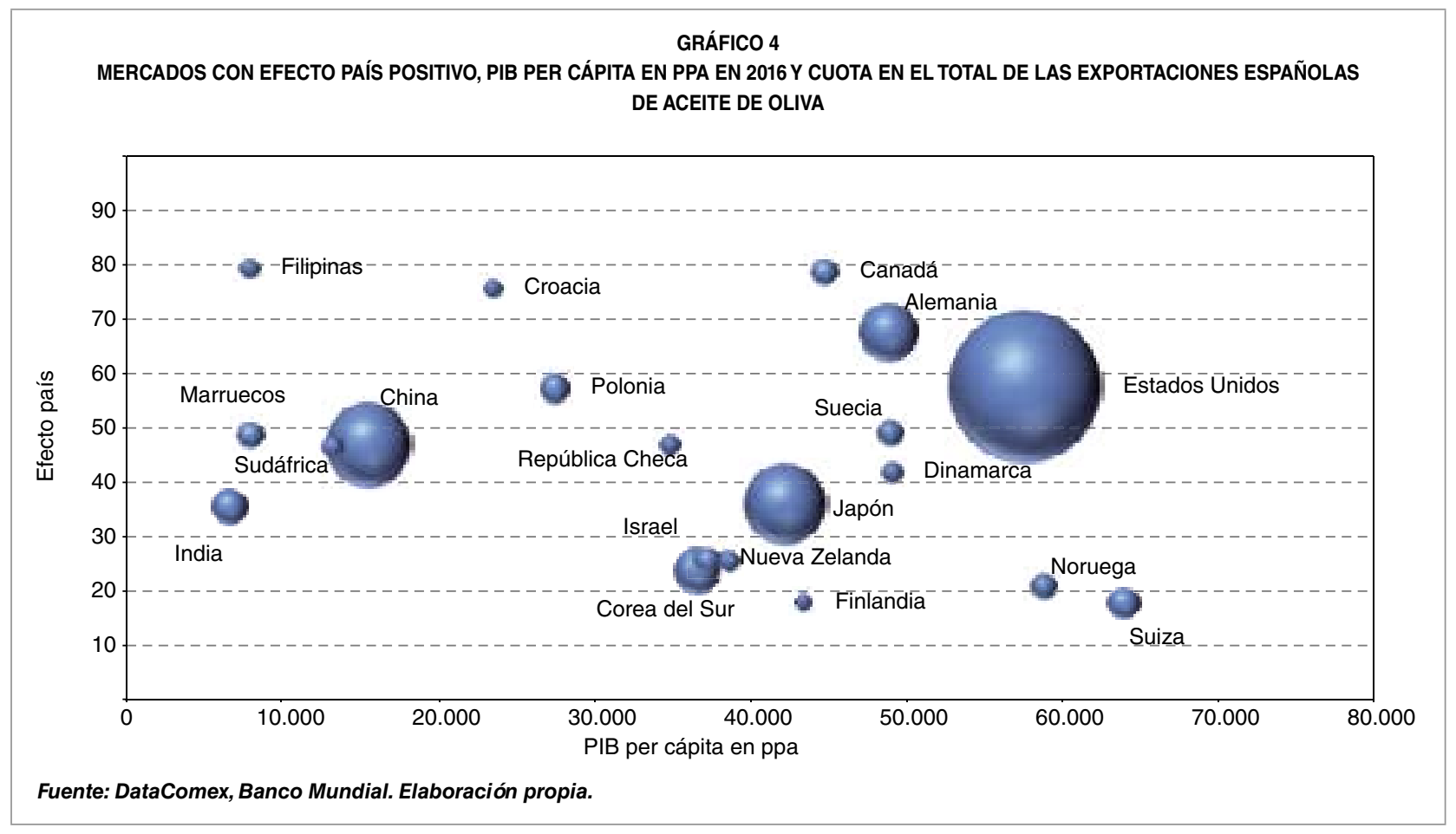

una variación media anual acumulada superior al 12 por 100 , entre el valor promedio 20132014 y 2016-2017, lo que debe animar a las compañías españolas a tratar de aprovechar la expansión que están experimentando estos mercados, auténticos responsables del crecimiento operado en los últimos años en las ventas de aceite de oliva español en el mercado mundial. En este agregado de países se pueden identificar distintas categorías. En concreto, se han establecido cuatro grupos considerando los tres parámetros representados en el Gráfico 3: efecto país, el PIB per cápita en ppa en 2016 y la cuota en el total de las exportaciones españolas de aceite de oliva.

En primer lugar, debido a su cercanía geográfica están los mercados europeos a consolidar, la mayoría miembros de la UE, en los que se incluyen Alemania, Suiza, Polonia, Noruega, Suecia, República Checa, Dinamarca, Finlandia y Croacia, a los que se uniría Israel, que presentan, en conjunto, bastante margen para la expansión de las exportaciones. En segundo lugar destacan las economías que presentan un elevado PIB per cápita, superior a 35.000 USD, que son destinos altamente desarrollados a consolidar, aunque más distantes geográfica y culturalmente, como es el caso de Estados Unidos, Canadá, Japón, Nueva Zelanda y Corea del Sur, siendo el primero de ellos el que mayor cuota de exportación concentra. En contraste con los anteriormente descritos se sitúa un tercer grupo, formado por China e India, dos destinos de gran potencial, con más de 1.300 millones de habitantes cada uno en 2016, con un PIB en paridad de poder adquisitivo conjunto que representa más del doble del PIB conjunto de Japón, Hong Kong, Singapur y Corea del Sur, que progresivamente van incrementando su peso en la economía mundial (Alcalá, Chorén y Fernández, 2012), en los que las exportaciones españolas tienen claras posibilidades de expansión y en los que se debe hacer valer las ventajas del sector oleícola. Por último, mercados de países en desarrollo de baja consolidación, pero en $\triangleright$ 
los que conviene estar presentes, como es el caso de Marruecos, Filipinas y Sudáfrica, que presentan un interés creciente por este producto español.

Los resultados que ofrece el análisis shift-share permiten afirmar que la selección de mercados de exportación del sector oleícola español afecta negativamente al incremento de sus ventas en el mercado internacional. Tres de los principales destinos, Italia, Francia y Portugal, que en 2017 concentran el 52,59 por 100 del total exportado, han protagonizado tasas de crecimiento para sus importaciones moderadas, influyendo en la evolución agregada de la variable nacional. Una situación que urge resolver, dado que es evidente que aquellos países que configuran la estructura de clientes internacionales del sector son responsables directos de la evolución de sus ventas en el extranjero. Un sector que dirige gran parte de su producción hacia destinos en los que la demanda de aceite de oliva está aumentando intensamente y que cuenta con importantes posibilidades para que sus exportaciones se incrementen de forma rápida.

\section{Consideraciones finales}

Para el sector oleícola nacional la exportación es una variable fundamental, a la que destina cada año una parte creciente de su producción, que supuso en 2015 más del 52 por 100 del total. Ante esta situación conviene aprovechar las oportunidades que ofrecen distintos mercados en los que la demanda está creciendo de forma intensa, de dentro y fuera de la UE, tal y como se desprende del análisis realizado en las páginas precedentes. Para ello, es necesario el desarrollo de una política comercial activa por parte del sector, capaz de aunar los intereses de la pequeña y la gran empresa exportadora, y que tenga presente los factores que a corto, medio y largo plazo van a influir en las pautas del mercado mundial de aceite de oliva, entre los que destacan los siguientes: (i) la generalización en los últimos años de prácticas comerciales proteccionistas, que generan incertidumbre y condicionan los flujos de comercio a nivel mundial; (ii) el aumento de la población, principal impulsor de los aumentos de la demanda de alimentos; (iii) el creciente proceso de urbanización de esta, que propicia la homogenización de las pautas de consumo; (iv) una mayor longevidad y envejecimiento de los ciudadanos, que influye en sus preferencias como demandantes; (v) el incremento del ingreso en las economías emergentes, su mayor nivel cultural, que está influyendo en sus conocimientos sobre nutrición y en la occidentalización de sus hábitos alimentarios y, por último, (vi) la digitalización de las relaciones comerciales y el uso del e-commerce. Variables económicas y sociales que tienen una gran influencia sobre lo que la población compra, sus preferencias por alimentos saludables, como el aceite de oliva, la manera en que los utilizan y los requisitos que exigen.

\section{Bibliografía}

[1] ALCALÁ, F.; CHORÉN, P. y FERNÁNDEZ, A. (2012). Patrones recientes de comercio y especialización internacional. Bilbao: Fundación BBVA.

[2] CONSEJO OLEÍCOLA INTERNACIONAL (2018). Cifras Aceites de oliva. Madrid: COI. Disponible en: http://www.internationaloliveoil.org/estaticos/view/131-world-olive-oil-figures?lang=es_ES (Recuperado: 2018, 1 de febrero).

[3] DONOSO, V. y MARTíN BARROSO, V. (2007). «Mercados de exportación de España: $D$ 
Francisco García Moral, Concepción Martínez Alcalá y Encarnación Moral Pajares

presente y futuro». Información Comercial Española, Revista de Economía, ‥ 836, pp. 41-58.

[4] DUNN, E.S. (1960). «A statistical and analytical technique for regional analysis». Papers and Proceedings of the Regional Science Association, n. $\circ$ 6, pp. 97-112.

[5] ICEX (2018). Perfil de la empresa exportadora española. Madrid: ICEX. Disponible en: https:// www.icex.es/icex/es/navegacion-principal/quees-icex/donde-estamos/red-territorial-de-comercio/navegacion-principallinformes-y-documentos $/ 4402144$.htm I ? id Comunidad $=7$ (Recuperado: 2018, 1 de febrero).

[6] MORAL, E. y LANZAS, J.R. (2008). «La exportación de aceite de oliva virgen en Andalucía: Dinámica y factores determinantes». Revista de Estudios Regionales, n.요 86, pp. 45-70.

[7] MORAL, E. y LANZAS, J.R. (2017). «Mercados prioritarios para el crecimiento de las ventas", en Gómez-Limón, J.A. y Parras-Rosa, M. (coords.). Economía y comercialización de los aceites de oliva. Factores y perspectivas para el liderazgo español del mercado global. Monografía 31, pp. 313-334. Almería: Cajamar.
[8] PERLOFF, H.S.; DUNN, E.S.; LAMPARD, E.E. y MUTH, R.F. (1960). Regions, Resources and Economic Growth. Baltimore:John Hopkins Press.

[9] PIEZAS-JERBI, N. y NEE, C. (2009). «Market shares in the post-Uruguay round era: A closer look using shift-share analysis». ERSD Staff Working Paper, n.․14, Ginebra: Organización Mundial de Comercio.

[10] SAYADI GMADA, S.; ERAACH, Y. y PARRALÓPEZ, C. (2017). «Signos de calidad diferenciada del aceite de oliva en Andalucía", en Gómez-Limón, J.A. y Parras-Rosa, M. (coords.). Economía y comercialización de los aceites de oliva. Factores y perspectivas para el liderazgo español del mercado global. Monografía 31, pp. 251-275. Almería: Cajamar.

[11] SEGUí, C. y MONSERRAT, A. (2008). «Evolución de la exportación catalana y composición de su crecimiento 1995-2005». Boletín Económico de ICE, n.․․ 2952, pp. 47-59.

[12] SUBDIRECCIÓN GENERAL DE COMERCIO INTERNACIONAL DE PRODUCTOS AGROALIMENTARIOS (2017). La balanza comercial agroalimentaria en 2016. Madrid: Subdirección General de Comercio Internacional de Productos Agroalimentarios. 